Rincón-Barón, E. J. Torres-Rodríguez, G. A., Passarelli, L. M., Zárate, D. A., Cuarán, V. L., \& Plata-Arboleda, S. (2021). Microsporogénesis y micromorfología del polen de la planta Alcea rosea (Malvaceae). Revista de Biología Tropical, 69(3), 852-864. https://doi.org/10.15517/rbt. v69i3.46936

\title{
Microsporogénesis y micromorfología del polen de la planta Alcea rosea (Malvaceae)
}

Edgar Javier Rincón-Barón ${ }^{1 *}$; (iD https://orcid.org/0000-0003-1347-171X

Gerardo Andrés Torres-Rodríguez ${ }^{2}$; (D) https://orcid.org/0000-0003-2381-2936

Lilian M. Passarelli³; (D https://orcid.org/0000-0002-8870-8622

Diego A. Zárate ${ }^{4}$; (D) https://orcid.org/0000-0001-9630-3927

Viviana Lucia Cuarán ${ }^{4}$; (D https://orcid.org/0000-0001-7295-0046

Sayonara Plata-Arboleda ${ }^{5}$; (D) https://orcid.org/0000-0001-8115-8798

1. Grupo de Investigación Agroambiente y Salud-MICROBIOTA, Facultad de Ciencias Médicas y de la Salud, Instituto de Investigación Masira, Universidad de Santander, Calle 70 No 55-210, Campus Universitario Lagos del Cacique, Bucaramanga, Colombia; ed.rincon@mail.udes.edu.co (*Correspondencia), ejrbaron@gmail.com

2. Unidad de Microscopía Electrónica, Universidad del Cauca, carrera 2 \# 1A-25 Museo de Historia Natural, Popayán, Cauca, Colombia; gator@unicauca.edu.co

3. Laboratorio de Estudios de Anatomía Vegetal Evolutiva y Sistemática (LEAVES), Facultad de Ciencias Naturales y Museo de La Plata, Universidad de la Plata, 64 entre 120 y diagonal 113, B1904 E, La Plata, Argentina; Impassarelli@yahoo.com.ar

4. Corporación Colombiana de Investigación Agropecuaria (AGROSAVIA), Centro de Investigación La Suiza, Km. 32, vía al mar, Vereda Galápagos, Rionegro, Santander, Colombia; dzarate@agrosavia.co, vcuaran@agrosavia.co

5. Instituto de Ciencias Biológicas, Universidad de Talca, Avenida Lircay S/N Talca, Chile; sayonara.plata@utalca.cl

Recibido 10-V-2021. Corregido 10-VII-2021. Aceptado 19-VIII-2021.

\section{ABSTRACT \\ Microsporogenesis and micromorphology of pollen grains of the plant Alcea rosea (Malvaceae)}

Introduction: Studies on microsporogenesis, micromorphology and structure of pollen grains in Malvaceae are scarce.

Objectives: To describe the process of microsporogenesis and micromorphological aspects of pollen grains in A. rosea.

Methods: Androphores were processed according to standard protocols for sectioning in paraffin. The obtained sections were stained with Safranin-Alcian blue, Aniline blue was used for immature and unfixed anthers and for resin sections of the androphores, Toluidine blue. Ultrathin sections were observed with transmission electron microscopy. For observation with scanning electron microscopy the material was fixed and dehydrated in 2.2 dimethoxypropane, dried to a critical point and coated with gold.

Results: Anthers differentiate from a cell mass at the distal ends of the stamen filaments. The wall of the mature anther presents an outer layer of epidermal cells and an inner layer, the endothecium. Microspore mother cells divide by mitosis and then undergo meiosis to form tetrads. The tapetum is initially cellular and forms a single layer of cells and then loses cellular integrity by invading the microsporangium locule, forming a periplasmodia, by the time the pollen grains are released it degenerated. During sporodermis formation, exine is first deposited and then intine. Pollen grains are pantoporate, apolar, with radial symmetry, spheroidal, with spines, bacula, granules and microgranules. Tectum is perforated with foveolae arranged homogeneously over the whole surface and pollenkit is present. Exine is broad and consists of a thick 3.5 to $4 \mu \mathrm{m}$ endexine and a thin ektexine (0.6-0.7 
$\mu \mathrm{m})$. The ultrastructure shows columellae forming the infratectum. Capitate glandular unicellular nectariferous trichomes covers the whole surface of the stamen filaments.

Conclusions: The structure and development of the anthers follows the known patterns for angiosperms. Simultaneous microsporogenesis and centripetal deposit of the sporodermis have been previously described for Malvaceae.

Key words: Malvaceae; microspore; palynology; pantoporate; sporodermis; tapetum; ultrastructure.

Alcea L. es un género de plantas perteneciente a la familia Malvaceae (Bayer \& Kubitzki, 2003) con aproximadamente 70 especies ampliamente distribuidas (Arabameri et al., 2020; Escobar-García et al., 2012). Es originaria de Asia y Europa y se tuvieron como centro de especiación las tierras altas de Turquía y Kurdistan (Escobar-García et al., 2012). El género Alcea ha sido considerado sinónimo del género Althaea L., sin embargo, estudios moleculares (Escobar-García et al., 2012) han corroborado la identidad de los dos géneros, tal y como lo han indicado los datos morfológicos (Bayer \& Kubitzki, 2003; Cabi et al., 2009; Uzunhisarcikli \& Vural, 2012).

$A$. rosea es una especie ampliamente cultivada y apreciada por su valor ornamental y medicinal, por estas razones ha sido introducida en los jardines de la mayor parte del mundo (Bayer \& Kubitzki, 2003; Johri \& Raghuvanshi, 2014; Judd \& Manchester, 1997). La especie está circunscrita a la subfamilia Malvoideae y se caracteriza por ser una hierba bianual o perenne de inflorescencia en racimo, flores hermafroditas protándricas, presencia de epicaliz de 6-8 segmentos connados en la región media. Los estambres son monadelfos y forman una columna estaminal o tubo llamado andróforo que se divide en múltiples filamentos que rematan cada uno en una antera monoteca (Li et al., 2012; Naskar \& Mandal, 2014; Uzunhisarcikli \& Vural, 2012). El ovario es policárpico y el estilo está protegido y atraviesa el centro del andróforo, los estigmas son numerosos y equivalentes al número de carpelos.

La morfología de los granos de polen de Alcea ha sido ampliamente estudiada, encontrándose variaciones locales en el tamaño, aunque la morfología y ornamentación de la esporodermis al parecer es conservada (Cabi et al., 2009; Shaheen et al., 2010). Aunque se ha descrito el proceso de microsporogénesis en algunas Malvaceae (Galati \& Rosenfeldt, 1998; Lattar et al., 2014), en esta especie permanece aún sin resolver. Conocer y entender los procesos de microsporogénesis permite dilucidar con mayor exactitud los procesos evolutivos y relaciones filogenéticas que se entretejen entre los diferentes grupos de plantas (Furness et al., 2002; Nadot et al., 2008). Atendiendo a estas razones en este trabajo de investigación se hace una descripción detallada del proceso de microsporogénesis, haciendo énfasis en la arquitectura y morfología de las células madre de las microsporas, formación y estructura de la pared de las anteras, descripción del patrón de microsporogénesis, formación del esporodermis, estructura y función del tapete, micromorfología y ultraestructura de los granos de polen. Así mismo, describe y detalla por primera vez la presencia de tricomas nectaríferos unicelulares capitados en los filamentos que sostienen las anteras de esta especie.

\section{MATERIALES Y MÉTODOS}

Las muestras de las anteras de $A$. rosea fueron recolectadas en un jardín privado de la escarpa occidental de la meseta de la ciudad de Bucaramanga, Santander, Colombia ( $7^{\circ} 07^{\prime} 44^{\prime \prime}$ $\left.\mathrm{N} \& 73^{\circ} 08^{\prime} 04^{\prime \prime} \mathrm{W}\right)$ a $916 \mathrm{~m}$ de altitud, durante los meses de junio-julio del 2020. Los especímenes de $A$. rosea fueron depositados en el herbario de la Universidad de Antioquia (HUA) con la referencia de recolección Rincón 024 . Se definieron varios estadios de maduración teniendo en cuenta el tamaño y desarrollo del botón floral. Se tomaron más de 10 plantas y de cada una de estas se tomaron 30 andróforos 
por cada etapa del desarrollo floral y estos se fijaron en una mezcla de formol, etanol y ácido acético (FAA) por 24-48 horas a $6{ }^{\circ} \mathrm{C}$. Posteriormente, se utilizaron andróforos completos o fragmentos de $1 \mathrm{~cm}$ de longitud para deshidratarlos en la serie gradual de etanol y dos pasos de aclaramiento en Xilol (Ruzin, 1999); estos incluyeron en Paraplast plus (Mc Cormick ${ }^{\circledR}$ ) durante 12 horas a $55^{\circ} \mathrm{C}$. Se obtuvieron secciones transversales y longitudinales con micrótomo rotatorio LEICA RM 2125, entre 4-5 $\mu \mathrm{m}$ de grosor. Se procedió a la tinción de las muestras como Azul de Alcian-Safranina (Ruzin, 1999; Demarco, 2017; Rincón et al., 2020). Para determinar la presencia de calosa y el patrón de microsporogénesis se hicieron montajes de las anteras inmaduras y sin fijar, con Azul de Anilina (Śnieżko, 2000).

Muestras adicionales de los andróforos en los diferentes momentos del desarrollo floral fueron fijadas en Glutaraldehído al $2.5 \%$ en buffer fosfato $0.2 \mathrm{M}$ pH 7.2 durante 24-48 horas a $6{ }^{\circ} \mathrm{C}$. Luego de la fijación, las muestras se lavaron en el mismo buffer seguido de agua destilada para posteriormente postfijarlas con Tetróxido de Osmio al $2 \%$ por 2 horas a $6{ }^{\circ} \mathrm{C}$ en oscuridad y agitación constante. Finalmente, las muestras se deshidrataron durante una hora en una serie gradual de etanol y durante 12 horas etanol al $100 \%$. Las muestras se embebieron en mezclas progresivas de óxido de propileno-resina Spurr, por una semana a temperatura ambiente. Se hicieron varios cambios de resina pura durante seis días en agitación constante. La resina fue polimerizada a $60{ }^{\circ} \mathrm{C}$ por 48 horas. Se obtuvieron secciones de 0.5 $0.7 \mu \mathrm{m}$ de grosor con cuchillas de vidrio en un Ultramicrótomo Leica Ultracut UCT $\AA$; las cuales se tiñeron con Azul de Toluidina (TBO) en bórax al $1 \%$, pH 3.6 por 30-60 segundos. Tanto las secciones en parafina como las de resina y las muestras sin fijar se examinaron con un microscopio fotónico Nikon eclipse $\mathrm{Ni}$ equipado con el sistema de contraste diferencial de interferencia (CDI) y aditamentos para fluorescencia, utilizando el programa NIS Elements versión 4.30 .02 de Nikon. Las muestras teñidas con Azul de anilina se observaron con filtro UV-2A (filtro de excitación 330-380 nm y filtro barrera $420 \mathrm{~nm}$ ) (Ruzin, 1999).

Así mismo y aplicando el procesamiento descrito anteriormente para la obtención de secciones en resina, se hicieron secciones ultrafinas (para microscopía electrónica de transmisión, MET) de la pared de los granos de polen de 70-80 nm de grosor con cuchilla de diamante. Estas secciones se contrastaron con acetato de uranilo y citrato de plomo durante $40 \mathrm{~min}$ y 5 min respectivamente y se observaron con un microscopio de transmisión JEOL JEM-101.

Para llevar a cabo las descripciones morfológicas con microscopía electrónica de barrido (MEB), las anteras maduras con los granos de polen fueron fijados y deshidratados en 2.2 Dimetoxipropano acidificado por 2 a 3 días, realizando un cambio de este solvente durante este tiempo (Halbritter et al., 2018; Rincón et al., 2021). Finalmente, se hicieron dos cambios en etanol absoluto durante 30 minutos cada uno, y procedió a desecar al punto crítico con un desecador SAMDRI®-795. Las muestras se montaron sobre cinta conductiva de carbono de doble cara y se recubrieron con oro en una ionizadora DENTON VACUUM DESK IV durante 10 minutos. Las observaciones y registro fotográfico se realizaron en un microscopio electrónico de barrido JEOL JSM-6490LV.

Para las descripciones de los granos de polen y las anteras se utilizaron los términos sensu Crang et al. (2018), Halbritter et al. (2018) y Punt et al. (2007). Las mediciones del tamaño de los granos de polen están representadas por la medida mínima, la media y medida máxima empleando un $\mathrm{N}=30$.

\section{RESULTADOS}

En el meristemo floral de $A$. rosea se aprecian los primordios androeciales que mediante divisiones mitóticas formará los filamentos estaminales, éstos soportarán a las anteras constituyendo en conjunto el andróforo (Fig. 1A). En los extremos de los filamentos estaminales en desarrollo se observa la presencia de una masa celular diferenciada, el tejido arqueospórico, formada por células con abundante 
citoplasma, un núcleo voluminoso que ocupa el centro de la célula y en éste se pueden apreciar uno o dos nucleolos. Estas células formarán a las células madre de las microsporas (Fig. 1B). Así mismo, a partir de células derivadas del tejido arquespórico también se formará la pared de las anteras, que inicialmente está formada por una sola capa parietal de células de contorno cuadrado y núcleo voluminoso que originará por sucesivas divisiones los distintos estratos de la pared de la antera (Fig. 1B).

Con el crecimiento y desarrollo, el andróforo se diferencia y en este se aprecian múltiples filamentos estaminales alargados que

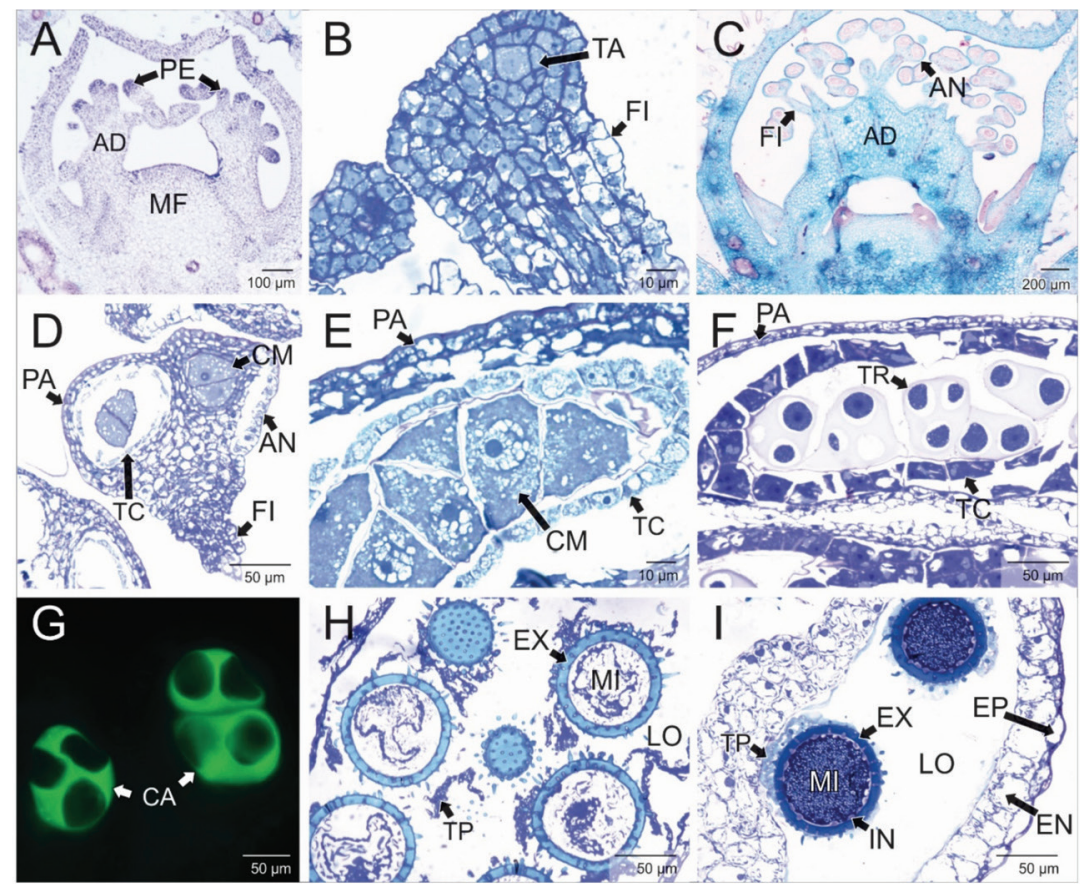

Fig. 1. Meristemo floral, andróforo y microsporogénesis de Alcea rosea. A. Primordios androeciales que formarán el andróforo (Azul de toluidina-TBO). B. Región apical del filamento estaminal. Se aprecia el tejido arquespórico que formará a las células madres de las microsporas (TBO). C. Andróforo en desarrollo. Se observan las anteras diferenciadas (SafraninaAzul de Alcian). D. Detalle de las anteras en maduración (TBO). E. Detalle de las células madre de las microsporas, tapete celular y pared de las anteras (TBO). F. Tétradas de microsporas. Se aprecia el tapete celular (TBO). G. Tétradas de microsporas. Se distinguen la cubierta de calosa (Filtro UV-2A, azul de Anilina). H-I. Microsporas en maduración y tapete plasmodial (TBO). En H se aprecia la exina gruesa y el tapete plasmodial en el lóculo microsporangial. En I se distinguen la exina y la intina y remanentes del tapete plasmodial, además de la epidermis y el endotecio. AD: andróforo; AN: anteras; TA: tejido arquespórico; CA: cubierta de calosa; CM: células madre de las microsporas; EX: exina; IN: Intina; FI: filamentos estaminales; LO: lóculo microsporangial; MF: meristemo floral; MI: microsporas; PA: pared de las anteras; PE: primordios androeciales; TC: tapete celular; TP: tapete plasmodium; TR: tétradas de microsporas.

Fig. 1. Floral meristem, androphore and microsporogenesis of Alcea rosea. A. Androecial primordia that will form the androphore (toluidine blue-TBO). B. Apical region of the staminal filament. Archesporic tissue that will form the microspore mother cells is appreciated (TBO). C. Androphore in development. Differentiated anthers are appreciated (Safranina-Alcian blue). D. Detail of maturing anthers (TBO). E. Detail of microspore mother cells, cellular tapetum and anther wall (TBO). F. Tetrads of microspores. Cellular tapetum is appreciated (TBO). G. Tetrads of microspores. Callose coated is appreciated (UV-2A filter, Aniline blue). H-I. Maturing microspores and plasmodial tapetum (TBO). In $\mathbf{H}$ the thick exine and the plasmodial tapetum can be seen in the microsporangial locule. In I exine and intine and remnants of the plasmodial tapetum are seen, in addition to the epidermis and endothecium. AD: androphore; AN: anthers; TA: archesporic tissue; CA: callose coated; CM: microspore mother cells; EX: exine; IN: intine; FI: staminal filament; LO: microsporangial locule; MF: floral meristem; MI: microspores; PA: anthers wall; PE: androecial primordia; TC: cellular tapetum; TP: plasmodial tapetum; TR: microspore tetrads. 
sostienen una antera diferenciada cada uno (Fig. 1C). Las anteras para este momento del desarrollo están formadas por una pared de varios estratos celulares y una capa de tapete celular que delimita a las células madre de las microsporas (Fig. 1D). Estas células madre son voluminosas con un núcleo excéntrico, en el cual se puede apreciar un prominente nucleolo y en su citoplasma se observan gran cantidad de gránulos de almidón que se aprecian traslúcidos ya que el TBO no los tiñe (Fig. 1D). A medida que la antera crece y se desarrolla, las células madre de las microsporas aumentan en número y la capa de tapete celular se hace más conspicua (Fig. 1E). Las células tapetales se aprecian de contorno cuadrangular o rectangular con un núcleo que ocupa la mayor parte del citoplasma y uno o dos nucleolos. La pared de la antera para este momento del desarrollo está formada por un estrato de tres capas celulares. La capa más externa que formará la epidermis presenta células de contorno entre cuadrangular a rectangular, en tanto que las demás capas internas son rectangulares, bajas o aplanadas (Fig. 1E).

Por medio de citocinesis meiótica simultánea las células madre de las microsporas forman tétradas de microsporas en disposición tetraédrica (Fig. 1F, Fig. 1G). Estas células se caracterizan por su contorno esférico con un núcleo voluminoso en posición central y abundantes gránulos de almidón (Fig. 1F). Las microsporas en la tétrada están cubiertas por una gruesa capa de calosa que se aprecian en color amarillo-verdoso al teñir con Azul de Anilina y observar con luz ultravioleta (Fig. $1 G)$. Una vez que las microsporas se separan entre sí, el tapete celular pierde la integridad celular y las células se fusionan formado un plasmodio que invade el lóculo de los microsporangios y rodea completamente a las microsporas (Fig. 1H). Para este momento del desarrollo, las microsporas han formado la exina. Con la maduración de las microsporas, se forma posteriormente la intina y el tapete plasmodial empieza a degenerar dejando libres a las microsporas en el lóculo microsporangial (Fig. 1I); en tanto que en la pared de la antera se aprecian dos estratos celulares uniestratificados, uno externo que corresponde a la epidermis y otro interno, el endotecio (Fig. 1I).

Una vez que la antera se abre, libera los granos de polen, estos se dispersan en mónades, son pantoporados, apolares, con simetría radial, esferoidales, grandes (110-116.5-127 $\mu \mathrm{m})$ (Fig. 2A). Presentan poros pequeños de 1 a $2 \mu \mathrm{m}$, con membrana delgada, y márgenes irregulares, separados por una distancia entre sí de alrededor de $10 \mu \mathrm{m}$ (Fig. 2B, Fig. 2C). La densidad de poros es en promedio 5 /campo de $20 \mu \mathrm{m}^{2}$. A través de estas aberturas se observa la presencia de una expansión de la intina, una estructura similar a una bácula pequeña, de 0.5 a $0.8 \mu \mathrm{m}$ (Fig. 2D). El téctum es perforado con ornamentación supratectal, presenta sobre su superficie diferentes elementos esculturales, algunos muy pequeños, microgránulos de entre 1 a $5 \mu \mathrm{m}$ y fovéolas dispuestas de forma homogénea sobre toda la superficie (Fig. 2D). El polenkit solo está sobre la superficie del téctum y no sobre los procesos ornamentales (Fig. 2B). La ornamentación más prominente está formada por espinas y báculas de mayor tamaño sin la base ensanchada o mamelones (Fig. 2B, Fig. 2C). Las espinas se ubican sobre la ectexina y también están constituidas por esporopolenina, en algunos casos se observa que la micrornamentación se interrumpe en la base de las espinas; estas son cónicas, rectas de extremos agudos o raramente truncados y a veces los extremos se curvan, tienen tono blanco destacándose del resto de la esporodermis que es gris (Fig. 2C). La exina está bien desarrollada, de entre 5-6 $\mu \mathrm{m}$ de grosor y está formada por una endexina muy gruesa ( 3.5 a 4 $\mu \mathrm{m})$ y una ectexina muy delgada $(0.6-0.7 \mu \mathrm{m})$, la intina también es gruesa y está formada por componentes de pared primaria $(0.2$ a $0.5 \mu \mathrm{m})$. El citoplasma de los granos de polen presenta abundantes gránulos de almidón distribuidos en toda su superficie (Fig. 2E). La ultraestructura muestra columelas claramente definidas formando el infratéctum (Fig. 2F).

La columna estaminal del andróforo es lisa y se divide en múltiples filamentos que sostienen las anteras (Fig. 2G). Estos filamentos 

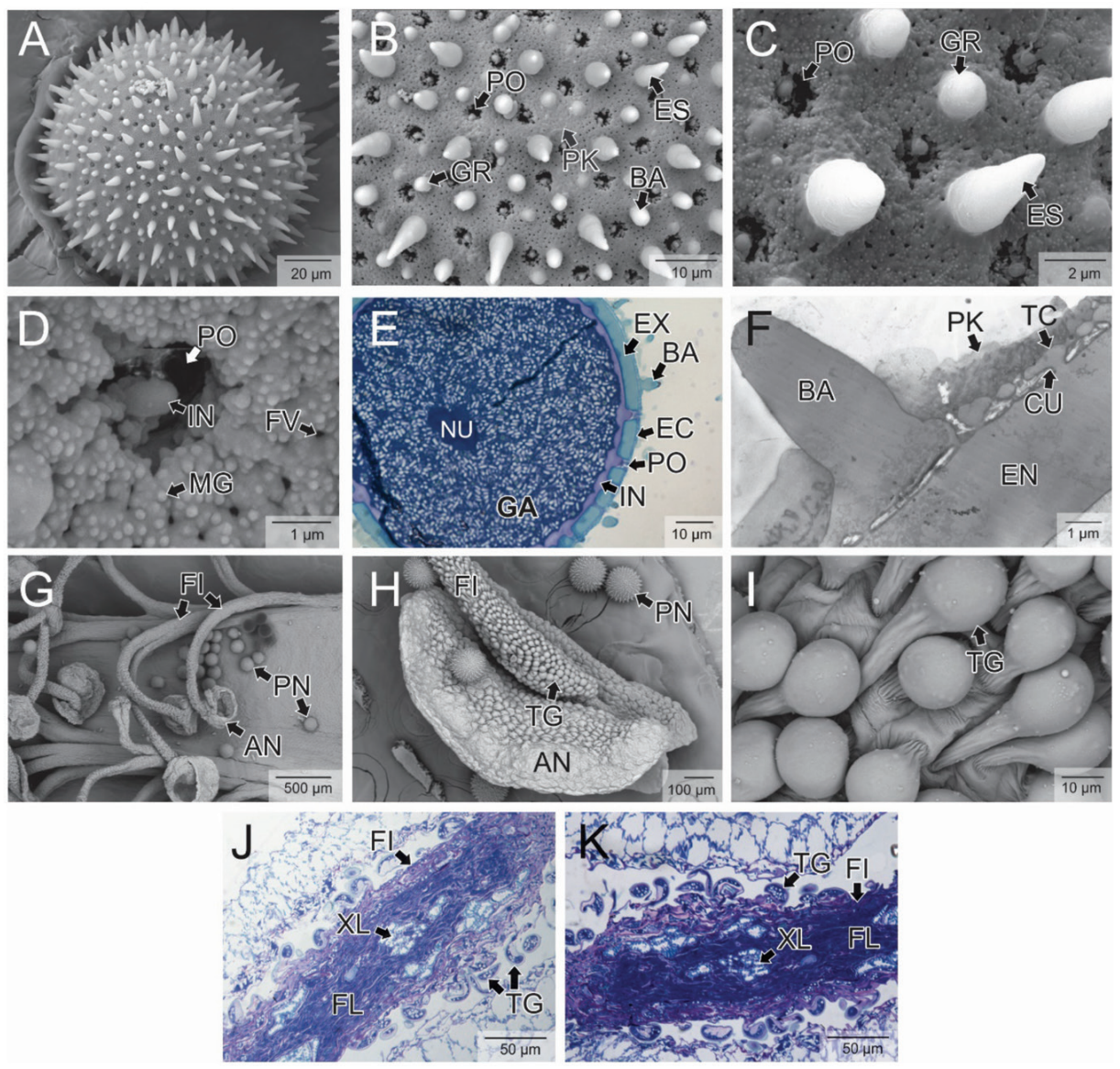

Fig. 2. Morfología y ultraestructura de los granos de polen y tricomas nectaríferos unicelulares glandulares capitados (TG) de Alcea rosea. A-C. Granos de polen (Microscopía electrónica de barrido-MEB). Se aprecia las espinas, báculas, gránulos, poros y polenkit. D. Detalle de un poro (MEB). Se observan los microgránulos y las foveolas. E. Sección trasversal del grano de polen (Azul de toluidina-TBO). Se indican los componentes de la esporodermis, núcleo y almidón en el citoplasma. F. Ultraestructura de la esporodermis en sección trasversal (Microscopía electrónica de transmisión-MET). G. Detalle del andróforo (MEB). Se aprecian los filamentos estaminales y las anteras. H-I Detalles de los filamentos estaminales (MEB). Se observan abundantes tricomas nectaríferos unicelulares glandulares capitados. J-K. Secciones longitudinales de los filamentos de las anteras (TBO). En K detalle histológico de los filamentos estaminales y de los TG. AD: andróforo; AN: anteras; BA: báculas; CU: columelas; EN: endexina; EC: ectexina; ES: espinas; FI: filamentos estaminales; FL: floema; FV: fovéolas; GA: gránulos de almidón; GR: gránulos; EX: exina; IN: intina; MG: microgránulos; NU: núcleo; PK: polenkit; PN: polen; PO: poros; TC: téctum; TG: tricomas nectaríferos unicelulares glandulares capitados; XL: xilema.

Fig. 2. Morphology and ultrastructure of pollen grains and capitate glandular unicellular nectariferous trichomes (TG) of Alcea rosea. A-C. Pollen grains (Scanning electron microscopy-SEM). Spines, bacula, granules, pores and pollenkit are appreciated. D. Detail of a pore (SEM). Microgranules and foveolae are observed. E. Cross section of pollen grain (toluidine blue-TBO). Components of the sporodermis, nucleus and starch in the cytoplasm are indicated. F. Ultrastructure of the sporodermis in cross section (Transmission electron microscopy-MET). G. Detail of the androphore (SEM). Stems filaments and anthers are appreciated. H-I. Stamen filaments details (SEM). Capitate glandular unicellular nectariferous trichomes are abundant. J-K. Longitudinal sections of stems filaments (TBO). In $\mathbf{K}$ histological detail of stems filaments and TG. AD: androphore; AN: anthers; BA: bacula; CU: columellae; EN: endexine; EC: ektexine; ES: spines; FI: stamen filaments; FL: phloem; FV: foveolae; GA: starch granules; GR: granules; EX: exine; IN: intine; MG: microgranules; NU: nucleus; PK: pollenkit; PN: pollen; PO: pores; TC: tectum; TG: capitate glandular unicellular nectariferous trichomes; XL: xylem. 
están recubiertos por tricomas nectaríferos unicelulares glandulares capitados (Fig. 2H, Fig. 2I), que están estrechamente asociados al haz vascular, en especial al floema (Fig. 2J, Fig. $2 \mathrm{~K})$. El floema está formado por una capa gruesa de varios estratos celulares que se tiñen intensamente de azul oscuro con TBO, en tanto que el xilema presenta menos estratos celulares (Fig. 2K). los tricomas nectaríferos presentan una cutícula gruesa y en su citoplasma se aprecian estructuras globulares translúcidas que no se tiñen con TBO y corresponden a abundantes gránulos de almidón (Fig. 2K).

\section{DISCUSIÓN}

En Malvaceae se ha indicado la formación de primordios androeciales en el meristemo floral que por crecimiento y diferenciación celular determinan la formación de la columna estaminal del andróforo y los filamentos estaminales (Von Balthazar et al., 2006). Estos filamentos presentan en sus ápices una masa de tejido arquespórico que se diferenciará para la formación de las células madre de las microsporas (Scott et al., 2004; Von Balthazar et al., 2006; Fernández et al., 2015), en A. rosea se observó una situación similar, lo que implicaría cierto grado de uniformidad ontogenética en el desarrollo y formación de estas estructuras. La diferenciación y desarrollo de las anteras de $A$. rosea muestra un patrón histológico característico de la mayoría de las Malvaceae (Tang et al., 2006; Lattar et al., 2012; Lattar et al., 2014), que implica la formación de pared de las anteras y células madre de las microsporas a partir de derivados celulares del tejido arquespórico; además, la pared de las anteras inicialmente está constituida de una sola capa celular y luego a medida que ésta madura se forman varios estratos celulares y al momento de la dehiscencia solo persiste la epidermis y el endotecio.

En Malvaceae, el tapete secretor es el más común (Tang et al., 2006; Lattar et al., 2014), aunque se han registrado en algunas especies de esta familia la presencia de tapete invasivo no sincitial (Galati et al., 2007; Lattar et al.,
2014) y también de tapete plasmodial (Strittmatter et al., 2000; Tang et al., 2009; Galati et al., 2011), en cualquiera de los casos, el tapete degenera para el momento de la liberación de los granos de polen, situación que se corroboró con el tapete plasmodial de $A$. rosea. Dado el estrecho contacto que se observó entre las microsporas de A. rosea y el tapete plasmodial, se apoya la idea de que el tapete facilita el proceso de maduración de las microsporas y de los granos de polen, así como, proporciona materia prima para la formación de la esporodermis, en especial de la exina (Pacini, 2010; Fernández et al., 2015).

La microsporogénesis en $A$. rosea es simultánea y se forman tétradas de microsporas en disposición tetraédrica, situación que es común en varios grupos de plantas incluida Malvaceae (Furness et al., 2002; Furness \& Rudall, 2004). Sin embargo, este patrón de microsporogénesis por lo general determina la formación de granos de polen con tres aberturas, que es el más común en eudicotiledóneas y se explica por los puntos de contacto de las microsporas en la tétrada (Furness \& Rudall, 2004). No obstante, los granos de polen de $A$. rosea son pantoporados, lo cual no se podría explicar por el modelo de contacto de las microsporas y al parecer está determinado por la presencia de acumulaciones regulares de calosa durante la formación de la tétrada (Prieu et al., 2019). Durante la microsporogénesis en plantas el depósito de la esporodermis es centrípeto, es decir, primero se deposita la exina formada principalmente de esporopolenina y posteriormente se forma la intina que está formada por componentes de pared primaria (Blackmore et al., 2007; Pacini \& Hesse, 2012). Este patrón de depósito de la esporodermis se verificó en $A$. rosea y se observó que la exina toma una tinción azul-turquesa por la presencia de esporopolenina cuando se tiñe con TBO, en tanto que la intina se tiñe de color violeta indicando la presencia de componentes de pared primaria, lo cual es congruente con trabajos anteriores que indican la utilidad de esta tinción diferencial para detectar estos compuestos en los granos de polen e incluso 
en las esporas de plantas (Rincón et al., 2019; Rincón et al., 2021).

Varios trabajos describen la morfología polínica en la familia Malvaceae s.l. y demuestran su condición euripalínica con diversidad de aberturas, estructura y escultura de la esporodermis (Bayer \& Kubitzki, 2003; Perveen \& Qaiser, 2009; Bibi et al., 2010; Hamdy \& Shamso, 2010; Saba \& dos Santos, 2015; Rincón et al., 2021); aunque el tipo pantoporado, con espinas de base mamelonada es el que caracteriza a las Malvaceae s.s. (Erdtman, 1986; Saba \& dos Santos, 2015) lo cual coincide con las observaciones hechas en este trabajo para $A$. rosea. Mas aun, algunos autores han tenido en cuenta la diversidad del polen para dilucidar la taxonomía y filogenia de la familia (Christensen, 1986; Culhane \& Blackmore, 1988), considerando a las Malvaceae s.s. como grupo monofilético y Bombacaceae, Sterculiaceae y Tiliaceae como parafiléticos (Cronquist, 1988; Judd \& Manchester, 1997; Whitlock, 2002).

La morfología palinológica de Alcea spp es descrita por varios autores (Cabi et al., 2009). Sin embargo, algunos caracteres como la ultraestructura de la pared, disposición de la intina y formación de las espinas no han sido aclarados. La morfología de la esporodermis es muy característica en la Tribu Malveae y la distingue de otras tribus, siendo la ectexina notablemente más delgada que la endexina como en Alcea y Bordasia (Christensen, 1986; Erdtman, 1986; Cuadrado, 2003), sin embargo, existen excepciones en algunos géneros de Malveae como Palaua spp, en el que no se observa esta diferencia en grosor y ambas capas son similares (Schneider et al., 2009).

En A. rosea las características del téctum con abundantes perforaciones junto al escaso grosor que posee la ectexina lo hace muy frágil y se observa que se disgrega fácilmente en las zonas aberturales. Sobre la esporodermis se deposita una delgada capa de polenkit que no cubre los procesos ornamentales supratectales, algo mencionado por Lunau et al., (2014), quienes explican además que la longitud de las espinas y el tamaño del grano en $A$. rosea entre otras especies, obstaculizan la recolección y el transporte del polen por insectos corbiculados, lo cual implica una defensa mecánica que minimiza el forrajeo y consumo de granos de polen. En contraste en esta investigación se observó que abejas del género Trigona juntan abundante cantidad de polen en las corbículas de sus patas (E. Rincón-Barón, observación personal, 15 de diciembre de 2020). Estas observaciones están acordes con la propuesta de que la morfología equinada de los granos de polen se relaciona estrechamente con la polinización de tipo entomófila (Sannier et al., 2009; Tanaka et al., 2004; Wang \& Dobritsa, 2018). Sin embargo, estudios recientes han indicado que ni el tamaño de los granos de polen, ni la micromorfología con la presencia de largas espinas, pueden explicar de forma certera la capacidad de recolección de los granos de polen por insectos corbiculados y proponen la importancia del polenkit en este aspecto (Konzmann et al., 2019); aunque A. rosea presenta polenkit, apoyar esta hipótesis necesitaría de más investigación.

En general la intina presenta engrosamientos a nivel de las aberturas o mantiene el grosor de la zona no abertural (Blackmore et al., 2007). Se documentó aquí en $A$. rosea, por medio de las diferentes tinciones y la observación con microscopía fotónica, una notoria prolongación de esta capa celulósica en los poros, zonas de germinación del tubo polínico, que podría ser mal interpretada como ornamentación de una membrana del poro. Esta característica fue observada por los autores en trabajos realizados con MET en otros géneros como Waltheria L. (Saba \& dos Santos, 2015) aunque no se menciona este rasgo. Es interesante, además, referir la presencia en el género Malvaceae citado un tipo novedoso de intina con estructura similar a columelas.

En cuanto a la ornamentación en Malvaceae está formada por procesos supratectales, sin embargo, éstos difieren según las tribus, en Malveae es heterogénea, se encuentran espinas, báculas y gemas de diferente tamaño, estas diferencias son poco frecuentes en otras tribus de la familia en la que los procesos son 
homogéneos (Christensen, 1986). En A. rosae se ha observado esta variación coincidiendo con El Naggar y Sawady (2008) y en contraposición a lo detallado por Culhane y Blackmore (1988) quienes señalan que las Malveae, y especialmente la especie citada no poseen diferencias siendo los procesos homogéneos.

Se observa en esta investigación, además, por primera vez, el grado menor de electrodensidad, con un tono más claro de los elementos ornamentales supratectales, estas características fueron advertidas por Cabi et al. (2009) al observar las figuras del polen de Alcea biennis Winterl y A. guestii Zohary en una investigación sobre Malvaceae de Turquía, aunque estos autores no hacen referencia a este detalle en su publicación. Esta característica de electrodensidad también ha sido descrita en otras angiospermas no emparentadas, como Canna spp (Ciciarelli et al., 2010) e indicarían una diferencia en la composición química de la esporopolenina que forma estos procesos. Durante la microsporogénesis los patrones de la exina son determinados durante la etapa en que la tétrada está recubierta por calosa (Barnes \& Blackmore, 1986; Blackmore \& Barnes, 1985; Blackmore \& Barnes, 1987), sin embargo, investigaciones bien documentadas sobre la formación de la pared, especialmente de las espinas, en Hibiscus syriacus L. demuestran que el desarrollo de estos elementos es posterior al estadio de tétrada, o sea no son homólogos a otros procesos de la exina (Takahashi \& Kouchi, 1988).

Los tricomas nectaríferos florales han sido registrados en algunos grupos de plantas (Vogel, 1997; Lopes et al., 2002; Stpiczyńska et al., 2018; Tölke et al., 2018; Tölke et al., 2019). Sin embargo, estos son característicos de la mayoría de las Malvaceae, en esta familia se ha indicado la presencia de tricomas nectaríferos multicelulares glandulares densamente agrupados en la superficie del cáliz, corola y algunas veces en la base del andróforo o androginóforo (Sawidis et al., 1987; Judd \& Manchester, 1997; Vogel, 2000; Bayer \& Kubitzki, 2003; Espolador-Leitão et al., 2005; Goldberg, 2009;
Lattar et al., 2009; Muneratto et al., 2014; Lattar et al., 2018). En contraste, en esta investigación se registra por primera vez la presencia de tricomas nectaríferos unicelulares glandulares capitados, densamente agrupados en los filamentos estaminales. La presencia de estas estructuras podría explicar la intensa actividad de los polinizadores sobre el andróforo de $A$. rosea (E. Rincón-Barón, observación personal, 15 de diciembre de 2020).

El estudio de la microsporogénesis y palinológico presentado aquí ha actualizado y ampliado la información conocida hasta el momento sobre $A$. rosea y sobre las Malvaceae en general, profundizando los datos relacionados con la ontogenia de las anteras, tétradas, microsporas, esporodermis y de la morfología y formación de los procesos supratectales, ornamentación y la ultraestructura de los granos de polen.

Declaración de ética: los autores declaran que todos están de acuerdo con esta publicación y que han hecho aportes que justifican su autoría; que no hay conflicto de interés de ningún tipo; y que han cumplido con todos los requisitos y procedimientos éticos y legales pertinentes. Todas las fuentes de financiamiento se detallan plena y claramente en la sección de agradecimientos. El respectivo documento legal firmado se encuentra en los archivos de la revista.

\section{AGRADECIMIENTOS}

Los autores agradecen a las siguientes instituciones y personas: a la Universidad de Santander (UDES) por el apoyo financiero y a la Universidad del Cauca (UNICAUCA) por su apoyo técnico e infraestructura. A la Corporación Colombiana de Investigación Agropecuaria (AGROSAVIA). A María Giomar Nates-Parra, Universidad Nacional de Colombia (UNAL) y a Alejandro Botero Galvis por la identificación del género de abeja que regularmente visita a $A$. rosea. 


\section{RESUMEN}

Introducción: Los estudios sobre microsporogénesis, micromorfología y estructura de los granos de polen en Malvaceae son escasos.

Objetivos: Describir el proceso de microsporogénesis y aspectos micromorfológicos de los granos de polen en $A$. rosea.

Métodos: Se procesaron más de 30 andróforos de acuerdo con los protocolos estándar para incrustar y seccionar en parafina. Las secciones obtenidas se tiñeron con Azul de Safranina-Alcian, las anteras inmaduras y no fijadas se tiñeron con Azul de anilina. Se procesaron secciones de resina adicionales de los andróforos y se tiñeron con azul de toluidina. Se observaron secciones ultrafinas con microscopía electrónica de transmisión (MET). Para la observación con microscopía electrónica de barrido (MEB), el material se fijó y deshidrató en 2,2 dimetoxipropano, luego se secó hasta un punto crítico y se recubrieron con oro.

Resultados: las anteras se diferencian de una masa celular en los extremos distales de los filamentos del estambre. La pared de la antera madura presenta una capa externa de células epidérmicas y una capa interna, el endotecio. Las células madre de microesporas se dividen por mitosis y luego experimentan meiosis para formar tétradas. El tapete es inicialmente celular y forma una sola capa de células y luego pierde integridad celular al invadir el lóculo de microsporangio, formando un periplasmodio. Durante la formación de la esporodermis, primero se deposita la exina y luego la intina. Para el momento de la liberación de los granos de polen, el tapete se ha degenerado por completo. Los granos de polen son pantoporados, apolares, con simetría radial, esferoidales, con espinas, báculas, gránulos y microgránulos. El téctum está perforado con fovéoleas dispuestas homogéneamente en toda la superficie y con polenkit. La exina es ancha $(5-6 \mu \mathrm{m})$ y consta de una endexina gruesa de 3.5 a $4 \mu \mathrm{m}$ y una ektexina fina $(0.6-0.7 \mu \mathrm{m})$. La ultraestructura muestra columelas claramente definidas formando el infratéctum. Se aprecian tricomas nectaríferos unicelulares glandulares capitados (TG) cubriendo toda la superficie de los filamentos de los estambres.

Conclusiones: La estructura y desarrollo de las anteras sigue los patrones conocidos de las angiospermas. La microsporogénesis simultánea y el depósito centrípeto de la esporodermis se han descrito previamente para Malvaceae.

Palabras clave: Malvaceae; microspora; palinología; pantoporado; esporodermis; tapete; ultraestructura.

\section{REFERENCIAS}

Arabameri, M., Khodayari, H., \& Zarre, S. (2020). Trichome micromorphology in Alcea L. and allied genera (Malvaceae) and its systematic implication. Nordic Journal of Botany, 38(6), 1-16.
Barnes, H., \& Blackmore, S. (1986). Some functional features in pollen development. En S. Blackmore, \& I. K. Ferguson (Eds.), Pollen and spores: form and function (pp. 71-80). Academic Press.

Bayer, C., \& Kubitzki, K. (2003). Malvaceae. En K. Kubitzki (Ed.), The families and genera of vascular plants (pp. 225-231). Springer-Verlag.

Bibi, N., Akhtar, N., Hussain, M., \& Khan, A. M. (2010). Systematic implications of pollen morphology in the family Malvaceae from North West frontier province, Pakistan. Pakistan Journal of Botany, 42(4), 2205-2214.

Blackmore, S., \& Barnes, S. H. (1985). Cosmos pollen ontogeny: a scanning electron microscope study. Protoplasma, 126(1-2), 91-99.

Blackmore, S., \& Barnes, S. H. (1987). Pollen wall morphogenesis in Tragopogon porrifolius L. (Compositae: Lactuceae) and its taxonomic significance. Review of Palaeobotany and Palynology, 52(2-3), 233-246.

Blackmore, S., Wortley, A. H., Skvarla, J. J., \& Rowley, J. R. (2007). Pollen wall development in flowering plants. New Phytologist, 174(3), 483-498.

Cabi, E., Başer, B., Uzunhisarcikli, M. E., \& Yavru, A. (2009). Pollen morphology of Alcea L. and Althaea L. genera (Malvaceae) in Turkey. Feddes Repertorium, 120(7-8), 405-418.

Christensen, P. B. (1986). Pollen morphological studies in the Malvaceae. Grana, 25(2), 95-117.

Ciciarelli, M. D. L. M., Passarelli, L. M., \& Rolleri, C. H. (2010). Morfología del polen en especies de Canna (Cannaceae) y su implicancia sistemática. Revista de Biología Tropical, 58(1), 63-79.

Crang, R., Lyons-Sobaski, S., \& Wise, R. (2018). Plant Anatomy: A Concept-Based Approach to the Structure of Seed Plants. Springer.

Cronquist, A. (1988). The evolution and classification of flowering plants ( $2^{\text {nd }}$ Ed.). New York Botanical Garden.

Cuadrado, G. (2003). Estudio Morfológico del Polen de Bordasia bicornis (Malvaceae). Bonplandia, 12(1-4), 137-140.

Culhane, K., \& Blackmore, S. (1988). Northwest European Pollen Flora, 41 Malvaceae. Review of Palaeobotany and Palynology, 57(1-2), 45-74.

Demarco, D. (2017). Histochemical analysis of plant secretory structures. In C. Pellicciari, \& M. Biggiogera (Eds.), Histochemistry of single molecules methods and protocols (pp. 313-330). Humana Press. 
El Naggar, S. M., \& Sawady, N. (2008). Pollen morphology of Malvaceae and its taxonomic significance in Yemen. Flora Mediterranea, 18, 431-439.

Erdtman, G. (1986). Pollen morphology and plant taxonomy. Angiosperms. Brill Verlag.

Escobar-García, P., Pakravan, M., Schönswetter, P., Aguilar, J. F., \& Schneeweiss, G. M. (2012). Phylogenetic relationships in the species-rich Irano-Turanian genus Alcea (Malvaceae). Taxon, 61(2), 324-332.

Espolador-Leitão, C. A., Strozi, R. M., Azevedo, A., de Araújo, J. M., Silva, K. L. F. \& García, C. R. (2005). Anatomy of the floral, bract, and foliar nectaries of Triumfetta semitriloba (Tiliaceae). Canadian Journal of Botany, 83(3), 279-286.

Fernández, G. J., Talle, B., \& Wilson, Z. A. (2015). Anther and pollen development: a conserved developmental pathway. Journal of Integrative Plant Biology, 57(11), 876-891.

Furness, C. A., \& Rudall, P. J. (2004). Pollen aperture evolution-a crucial factor for eudicot success? Trends in Plant Science, 9(3), 154-158.

Furness, C. A., Rudall, P. J., \& Sampson, F. B. (2002). Evolution of microsporogenesis in angiosperms. International Journal of Plant Sciences, 163(2), 235-260.

Galati, B. G., Gotelli, M. M., Rosenfeldt, S., Torretta, J. P., \& Zarlavsky, G. (2011). Orbicules in relation to the pollination modes. In B. J. Kaiser (Ed.), Pollen: structure, types and effects (pp. 1-15). Nova Science Publisher.

Galati, B. G., Monacci, F., Gotelli, M. M., \& Rosenfeldt, S. (2007). Pollen, tapetum and orbicule development in Modiolastrum malvifolium (Malvaceae). Annals of Botany, 99(4), 755-763.

Galati, B. G., \& Rosenfeldt, S. (1998). The pollen development in Ceiba insignis (Kunth) Gibbs and Semir ex Chorisia speciosa St Hil. (Bombacaceae). Phytomorphology, 48(2), 121-129.

Goldberg, L. (2009). Patterns of nectar production and composition, and morphology of floral nectaries in Helicteres guazumifolia and Helicteres baruensis (Sterculiaceae): two sympatric species from the Costa Rican tropical dry forest. Revista de Biología Tropical, 57(Suppl. 1), 161-177.

Halbritter, H., Ulrich, S., Grimsson, F., Weber, M., Zetter, R., Hesse, M., Buchner, R., Svojtka, M., \& FroschRadivo, A. (2018). Illustrated pollen terminology $\left(2^{\text {nd }}\right.$ Ed.). Springer.

Hamdy, R., \& Shamso, E. (2010). Pollen morphology of Sterculiaceae (s. str.) in Egypt and its taxonomic significance. Egyptian Journal of Botany, 50, 103-117.
Johri, A., \& Raghuvanshi, R. K. (2014). Floral biology, pollination and breeding system in Alcea rosea (L.) syn. Althaea chinensis Wall. (Malvaceae). The International Journal of Plant Reproductive Biology, 6(2), 139-144.

Judd, W. S., \& Manchester, S. R. (1997). Circumscription of Malvaceae (Malvales) as determined by a preliminary cladistic analysis of morphological, anatomical, palynological, and chemical characters. Brittonia, 49(3), 384-405.

Konzmann, S., Koethe, S., \& Lunau, K. (2019). Pollen grain morphology is not exclusively responsible for pollen collectability in bumble bees. Scientific Reports, 9(1), 1-8.

Lattar, E. C., Galati, B. G., Carrera, C. S., \& Ferrucci, M. S. (2018). Floral nectaries of Heliocarpus popayanensis and Luehea divaricata (Malvaceae-Grewioideae): structure and ultrastructure. Australian Journal of Botany, 66(1), 59-73.

Lattar, E. C., Galati, B. G., \& Ferrucci, M. S. (2012). Ultrastructural study of pollen and anther development in Luehea divaricata (Malvaceae, Grewioideae) and its systematic implications: Role of tapetal transfer cells, orbicules and male germ unit. FloraMorphology, Distribution, Functional Ecology of Plants, 207(12), 888-894.

Lattar, E. C., Galati, B. G., \& Ferrucci, M. S. (2014). Comparative study of anther development, microsporogenesis and microgametogenesis in species of Corchorus, Heliocarpus, Luehea and Triumfetta (Malvaceae: Grewioideae) from South America. New Zealand Journal of Botany, 52(4), 429-445.

Lattar, E. C., Solís, S. M., Avanza, M. M., \& Ferrucci, M. S. (2009). Estudios morfo-anatómicos en nectarios florales y extraflorales de Triumfetta rhomboidea (Malvaceae, Grewioideae). Boletín de la Sociedad Argentina de Botánica, 44(1-2), 33-41.

Li, Q., Ruan, C. J., Teixeira da Silva, J. A., \& Wang, X. Y. (2012). Floral morphology and mating system of Alcea rosea (Malvaceae). Plant Ecology and Evolution, 145(2), 176-184.

Lopes, A. V., Vogel, S., \& Machado, I. C. (2002). Secretory trichomes, a substitutive floral nectar source in Lundia A. DC. (Bignoniaceae), a genus lacking a functional disc. Annals of Botany, 90(2), 169-174.

Lunau, K., Piorek, V., Krohn, O., \& Pacini, E. (2014). Just spines-mechanical defense of malvaceous pollen against collection by corbiculate bees. Apidologie, 46(2), 144-149.

Muneratto, J. C., De Souza, L. A., \& De Almeida, O. J. G. (2014). The floral structure of three weedy species of Sida (Malvaceae). Journal of the Botanical Research Institute of Texas, 8(1), 127-137. 
Nadot, S., Furness, C. A., Sannier, J., Penet, L., Triki-Teurtroy, S., Albert, B., \& Ressayre, A. (2008). Phylogenetic comparative analysis of microsporogenesis in angiosperms with a focus on monocots. American Journal of Botany, 95(11), 1426-1436.

Naskar, S., \& Mandal, R. (2014). Characterization of some common members of the family Malvaceae ss on the basis of morphology of selective attributes: epicalyx, staminal tube, sigmatic head and trichome. Indian Journal of Plant Sciences, 4(3), 79-86.

Pacini, E. (2010). Relationships between tapetum, loculus, and pollen during development. International Journal of Plant Sciences, 171(1), 1-11.

Pacini, E., \& Hesse, M. (2012). Uncommon pollen walls: reasons and consequences. Verhandlungen der Zoologisch-Botanischen Gesellschaft in Osterreich, 148, 291-306.

Perveen, A., \& Qaiser, M. (2009). Pollen flora of PakistanMalvaceae: Dombeyoideae-Lxii. Pakistan Journal of Botany, 41(2), 491-494.

Prieu, C., Toghranegar, Z., Gouyon, P. H., \& Albert, B. (2019). Microsporogenesis in angiosperms producing pantoporate pollen. Botany Letters, 166(4), 457-466.

Punt, W., Hoen, P. P., Blackmore, S., Nilsson, S., \& Le Thomas, A. (2007). Glossary of pollen and spore terminology. Review of Palaeobotany and Palynology, 143(1-2), 1-83.

Rincón, B. E. J., Grisales, E. C., Cuaran, V. L., \& Cardona, N. L. (2020). Alteraciones anatómicas e histoquímicas ocasionadas por la oidiosis en hojas de Hydrangea macrophylla (Hydrangeaceae). Revista de Biología Tropical, 68(3), 959-976.

Rincón, B. E. J., Guerra, S. B. E., Restrepo, Z. D. E., \& Espinosa, M. S. (2019). Ontogenia e histoquímica de los esporangios y escamas receptaculares del helecho epífito Pleopeltis macrocarpa (Polypodiaceae). Revista de Biología Tropical, 67(6), 1292-1312.

Rincón, B. E. J., Zarate, D. A., Agudelo, G. A., Cuarán, V. L., \& Passarelli, L. M. (2021). Micromorfología y ultraestructura de las anteras y los granos de polen en diez genotipos élite de Theobroma cacao (Malvaceae). Revista de Biología Tropical, 69(2), 403-421.

Ruzin, S. E. (1999). Plant microtechnique and microscopy. Oxford University.

Saba, M. D., \& dos Santos, F. D. A. R. (2015). Pollen morphology and exine ultrastructure of selected species of Waltheria L. (Byttnerioideae-Malvaceae). Review of Palaeobotany and Palynology, 221, 204-210.

Sannier, J., Baker, W. J., Anstett, M. C., \& Nadot, S. (2009). A comparative analysis of pollinator type and pollen ornamentation in the Araceae and the
Arecaceae, two unrelated families of the monocots. BMC Research Notes, 2(1), 1-11.

Sawidis, T. H., Eleftheriou, E. P., \& Tsekos, I. (1987). The floral nectaries of Hibiscus rosa-sinensis: 1. development of the secretory hairs. Annals of Botany, 59(6), 643-652.

Schneider, J. V., Klie, D., Kacza, J., \& Huertas, M. L. (2009). Infrageneric variability of pollen morphology in Palaua (Malveae, Malvaceae) and the taxonomic utility of quantitative pollen characters. Grana, 48(4), 258-269.

Scott, R. J., Spielman, M., \& Dickinson, H. G. (2004). Stamen structure and function. The Plant Cell, 16(S1), S46-S60

Shaheen, N., Khan, M. A., Yasmin, G., Hayat, M. Q., Munsif, S., \& Ahmad, K. (2010). Foliar epidermal anatomy and pollen morphology of the genera Alcea and Althaea (Malvaceae) from Pakistan. International Journal of Agriculture and Biology, 12(3), 329-334.

Śnieżko, R. (2000). Fluorescence microscopy of aniline blue stained pistils. In W. V. Dashek (Ed.), Methods in plant electron microscopy and cytochemistry (pp. 81-86). Humana Press.

Stpiczyńska, M., Płachno, B. J., \& Davies, K. L. (2018). Nectar and oleiferous trichomes as floral attractants in Bulbophyllum saltatorium Lindl. (Orchidaceae). Protoplasma, 255(2), 565-574.

Strittmatter, L. I., Galati, B. G., \& Monacci, F. (2000). Ubisch bodies in the peritapetal membrane of $\mathrm{Abu}$ tilon pictum Gill (Malvaceae). Beiträge zur Biologie der Pflanzen, 71, 1-10.

Takahashi, M., \& Kouchi, J. (1988). Ontogenetic development of spinous exine in Hibiscus syriacus (Malvaceae). American Journal of Botany, 75(10), 1549-1558.

Tanaka, N., Uehara, K., \& Murata, J. (2004). Correlation between pollen morphology and pollination mechanisms in the Hydrocharitaceae. Journal of Plant Research, 117(4), 265-276.

Tang, Y. A., Gao, H. U. I., Wang, C. M., \& Chen, J. Z. (2006). Microsporogenesis and microgametogenesis of Excentrodendron hsienmu (Malvaceae sl) and their systematic implications. Botanical Journal of the Linnean Society, 150(4), 447-457.

Tang, Y. A., Gao, H., \& Xie, J. Z. (2009). An embryological study of Eriolaena candollei Wallich (Malvaceae) and its systematic implications. Flora, 204(8), 569-580.

Tölke, E. D., Bachelier, J. B., Lima, E. A., Galetto, L., Demarco, D., \& Carmello-Guerreiro, S. M. (2018). Diversity of floral nectary secretions and structure, and implications for their evolution in Anacardiaceae. 
Botanical Journal of the Linnean Society, 187(2), 209-231.

Tölke, E. D., Capelli, N. V., Pastori, T., Alencar, A. C., Cole, T. C. H., \& Demarco, D. (2019). Diversity of floral glands and their secretions in pollinator attraction. In J. M. Mérillon, \& K. G. Ramawat (Eds.), Co-Evolution of Secondary Metabolites (pp.1-46). Reference series in phytochemistry. Springer.

Uzunhisarcikli, M. E., \& Vural, M. (2012). The taxonomic revision of Alcea and Althaea (Malvaceae) in Turkey. Turkish Journal of Botany, 36(6), 603-636.

Vogel, S. (1997). Remarkable nectaries: structure, ecology, organophyletic perspectives I. Substitutive nectaries. Flora, 192(4), 305-333.
Vogel, S. (2000). The floral nectaries of Malvaceae sensu lato-a conspectus. Kurtziana, 28(2), 155-171.

Von Balthazar, M., Schönenberger, J., Alverson, W. S., Janka, H., Bayer, C., \& Baum, D. A. (2006). Structure and evolution of the androecium in the Malvatheca clade (Malvaceae sl) and implications for Malvaceae and Malvales. Plant Systematics and Evolution, 260(2), 171-197.

Wang, R., \& Dobritsa, A. A. (2018). Exine and aperture patterns on the pollen surface: Their formation and roles in plant reproduction. Annual Plant Reviews, $1,1-40$

Whitlock, B. A. (2002). Malvales. Encyclopedia of Life Science. John Wiley \& Sons Eds. 